\title{
Antimicrobial consumption on dairy herds and its association with antimicrobial inhibition zone diameters of non-aureus staphylococci and Staphylococcus aureus isolated from subclinical mastitis
}

\author{
M. Stevens, ${ }^{* 1}$ S. Piepers, ${ }^{*}$ K. Supré, $†$ and S. De Vliegher* \\ ${ }^{*}$ M-team and Mastitis and Milk Quality Research Unit, Department of Reproduction, Obstetrics and Herd Health, Faculty of Veterinary Medicine, \\ Ghent University, 9820 Merelbeke, Belgium \\ †Milk Control Centre Flanders, 2500 Lier, Belgium
}

\begin{abstract}
The main objectives of this study were to quantify the consumption of antimicrobials on a convenience sample of dairy herds and to determine the association between herd-level antimicrobial consumption and inhibition zone diameters (IZD) of non-aureus staphylococci and Staphylococcus aureus isolates from subclinical mastitis cases. Also, the association between the IZD of nonaureus staphylococci and Staph. aureus isolates within a herd was studied. Antimicrobial consumption data on 56 Flemish dairy farms were obtained between 2013 and 2014 by so-called garbage can audits and expressed as antimicrobial treatment incidence (ATI), with the unit of ATI being the number of defined daily doses animal (DDDA) used per 1,000 cow-days. The average total ATI in adult dairy cattle for all active substances was 18.73 DDDA per 1,000 cow-days and ranged from 6.28 to 42.13 DDDA between herds. The ATI of critically important (for human health) antimicrobials was 6.91 DDDA per 1,000 cow-days; that is, $37 \%$ of total antimicrobial consumption. The average ATI for intramammary therapy of (sub)clinical mastitis, intramammary dry-cow therapy, and systemically administered therapy was 5.20, 6.70, and 6.73 DDDA, respectively. The IZD of 239 non-aureus staphylococci and 88 Staph. aureus isolates originating from milk samples from cows with subclinical mastitis collected on selected dairy herds were determined using Kirby-Bauer disk diffusion and ranged between 6 and $42 \mathrm{~mm}$. Because only a limited number of clinical breakpoints (Clinical and Laboratory Standards Institute) and epidemiological cut-off values (European Committee on Antimicrobial Susceptibility Testing) are available for mastitis-causing bacteria in bovine, IZD were used as a proxy for antimicrobial resistance. Inhibition zone diameters of
\end{abstract}

Received June 26, 2017.

Accepted November 27, 2017.

${ }^{1}$ Corresponding author: Marina.Stevens@Ugent.be non-aureus staphylococci for cefquinome, a critically important $\beta$-lactam antibiotic, were negatively associated with the ATI of critically important $\beta$-lactam for systemically administered therapy and positively with the ATI for intramammary therapy of (sub)clinical mastitis of critically important $\beta$-lactam antimicrobials. Only for neomycin was a positive association between the IZD of non-aureus staphylococci and Staph. aureus isolates within the same herd observed.

Key words: antimicrobial consumption, staphylococci, subclinical mastitis, inhibition zone diameters

\section{INTRODUCTION}

Acquired antimicrobial resistance is a growing global concern in both human and veterinary medicine because it can result in treatment failure. The 2 main factors involved in the development of antimicrobial resistance in bacteria are the presence of resistance genes and selection pressure caused by the use of antimicrobials (Levy, 1997; Acar and Moulin, 2006; Silbergeld et al., 2008; Chantziaras et al., 2014). In dairy cattle, most antimicrobials are being used for the prevention and control of mastitis (Mitchell et al., 1998; Pol and Ruegg, 2007b; Menéndez González et al., 2010; Stevens et al., 2016). Potential associations between antimicrobial consumption on dairy herds and antimicrobial susceptibility of bovine mastitis pathogens have been studied, but not in Europe and not in much detail (Rajala-Schultz et al., 2004; Pol and Ruegg, 2007a; Saini et al., 2012b).

Over the last $10 \mathrm{yr}$, non-aureus staphylococci, also referred to as CNS, have become the most common cause of subclinical mastitis worldwide (Chaffer et al., 1999; Rajala-Schultz et al., 2004; Piepers et al., 2007; Vanderhaeghen et al., 2014; Sztachańska et al., 2016), particularly in heifers (De Vliegher et al., 2012). They form a heterogeneous group of more than 50 species, of which approximately 10 have been associated with IMI in dairy cattle (Vanderhaeghen et al., 2014) and are abundantly present in bulk milk (De Visscher et al., 2017). Non-aureus staphylococci are also present in 
other bovine-associated habitats such as air, housing, bedding material, milking equipment, and body parts such as teat apices, hair coat, nares, and the vagina (White et al., 1989; Nagase et al., 2002; Paduch and Kroemker, 2011; Piepers et al., 2011; Piessens et al., 2012; Braem et al., 2013; De Visscher et al., 2014, 2016). Their omnipresence, especially on the body of the cows, implies that non-aureus staphylococci are generally more exposed to antimicrobial treatments than other mastitis pathogens, which could explain the higher levels of antimicrobial resistance of non-aureus staphylococci compared with the more pathogenic Staphylococcus aureus (Owens and Watts, 1988; Taponen and Pyorala, 2009; Schmidt et al., 2015). It has been suggested that non-aureus staphylococci might act as a potential reservoir for resistance genes that can be transferred and integrated into the genome of Staph. aureus (Holmes and Zadoks, 2011; Otto, 2013; Vitali et al., 2014).

One of the most widely used methods for measuring antimicrobial susceptibility of bacteria is the KirbyBauer disk diffusion test, in which radial zones of bacterial growth inhibition are cross-referenced to breakpoints to determine whether an organism is susceptible or resistant to therapy with a particular antimicrobial drug (Traub and Leonhard, 1994). Still, the required clinical breakpoints to correctly interpret the results are often unavailable for a specific combination of pathogen and antimicrobial per host species (CLSI, 2008, 2015; http://www.eucast.org/ast_of_veterinary_pathogens/ ). This is the case particularly for non-aureus staphylococci isolated from bovine milk, partly because they are generally considered minor pathogens and typically not treated with antimicrobials. Using inhibition zone diameters (IZD) for non-aureus staphylococci and for Staph. aureus to determine the presence of (acquired) genetic resistance elements as a proxy for potential antimicrobial resistance, rather than relying on (clinical) breakpoints, avoids these issues.

The main objectives of this study were (1) to quantify the use of antimicrobials on a convenience sample of dairy herds, (2) to determine the association between the herd-level antimicrobial consumption and the IZD of non-aureus staphylococci and Staph. aureus isolates, respectively, and (3) to determine the association between the IZD of the non-aureus staphylococci and Staph. aureus isolates originating from the same herds.

\section{MATERIALS AND METHODS}

\section{Herds and Cows}

Data collected for this study originated from a convenience sample of 56 Flemish dairy herds. Herd inclusion criteria were (1) participation in the DHI program in Flanders with an interval of 4 to 6 wk between 2 testdays, and (2) record keeping of all treatments in adult cattle (date of occurrence, identification number of animal, treatment protocol applied). More details about the included herds are described elsewhere (Stevens et al., 2016).

From March to April 2014, approximately 10 lactating cows per herd with subclinical mastitis (defined as a test-day SCC $>150,000$ cells $/ \mathrm{mL}$ and $>250,000$ cells/ $\mathrm{mL}$ for heifers and multiparous cows, respectively) were randomly selected for milk sampling for bacteriological culturing.

\section{Antimicrobial Consumption Data}

Antimicrobial consumption data were retrieved by so-called garbage can audits, as described in a previous paper reporting on the antimicrobial consumption of the herds between January 2012 and February 2013 (Stevens et al., 2016). For the current paper, empty antimicrobial receptacles collected from March 2013 to February 2014 were used. Product name, volume, and number of receptacles were registered using a datasheet that included herd identification number and start and end date of collection for that particular herd. The quantification of the herd-level antimicrobial consumption was defined by the antimicrobial treatment incidence (ATI) as described previously (Stevens et al., 2016) and expressed as the number of defined daily doses animal (DDDA) used per 1,000 cows per day.

\section{Classification of Antimicrobials}

Route of Administration and Indication. Antimicrobials registered for intramammary use were divided into intramammary injectors registered for dry-cow therapy and injectors registered for the intramammary treatment of (sub)clinical mastitis cases. All systemically injectable antimicrobials used for the therapy of various diseases (including mastitis) are hereafter referred to as "systemically administered therapy." Intrauterine use of antimicrobials was very limited and therefore not taken into account in further analyses, as was done previously (Stevens et al., 2016).

Strata of Antimicrobials. Four strata were generated based on classification as $\beta$-lactam and non- $\beta$-lactam and their importance in human health: critically important (i.e., third- and fourth-generation cephalosporins and fluoroquinolones) or not critically important, according to the Office International des Épizooties (OIE) classification: stratum 1: critically important $\beta$-lactam antimicrobials (i.e., third- and fourth-generation cephalosporins); stratum 2: critically 
important non- $\beta$-lactam antimicrobials (i.e., fluoroquinolones); stratum 3: not critically important $\beta$-lactam antimicrobials (i.e., aminopenicillins, aminopenicillinclavulanic acid combination, aminopenicillin-polymyxin combination, first-generation cephalosporins, first-generation cephalosporin-aminoglycoside combination, penicillins, penicillin-aminopenicillin combination, and the penicillin-aminoglycoside combination); and stratum 4: not critically important non- $\beta$-lactam antimicrobials (i.e., aminoglycosides, lincosamides, lincosamide-aminoglycoside combination, macrolides, polymyxins, rifaximins, sulfonamide-trimethoprim combination, and tetracyclines).

\section{Collection of Milk Samples}

Quarter foremilk samples were collected aseptically by either the farmer or the first author and colleagues of the research group at milking (or between successive milkings on herds equipped with an automatic milking system) following the guidelines of the National Mastitis Council (Oliver et al., 2004). Sample materials were provided to the farmers and (aseptic) sampling procedures were explained. Sampling date, cow identification number, and quarter position were recorded. The quarter milk samples were transported under cooled conditions to the Flemish Milk Control Centre (MCC, Lier, Belgium), where routine bacteriological culturing was performed.

\section{Bacteriological Culturing}

Bacteriological culturing was performed following the National Mastitis Council guidelines (NMC, 1999). From each sample, $10 \mu \mathrm{L}$ of milk was spread on a quadrant of an esculin blood agar plate (Oxoid, Basingstoke, UK) and incubated aerobically at $37^{\circ} \mathrm{C}$ for 24 to $48 \mathrm{~h}$. Samples were considered to be culture-positive if one or more colonies were observed $(\geq 100 \mathrm{cfu} / \mathrm{mL})$. Identification of bacteria was done by Gram staining, inspection of colony morphology, and biochemical testing. The catalase test was used to distinguish staphylococci (positive reaction) from the Streptococcus-Enterococcus group (negative reaction). Staphylococcus aureus were differentiated from non-aureus staphylococci by colony morphology, hemolysis patterns, and DNase tests. A DNase test was considered positive if the clearing zone was 3 times the width of the incubation streak and negative/intermediate positive when the zone was less than 3 times the width of the streak. An isolate was considered as Staph. aureus when showing a complete or double hemolytic pattern after $48 \mathrm{~h}$ of incubation and a positive DNase test. All other catalase-positive isolates (thus, isolates showing no or single hemolysis and a negative/intermediate DNase test) were defined as "non-aureus staphylococcus"). Testing of DNase activity has a sensitivity of $97 \%$ and a specificity of $83 \%$ after a 24-h incubation for Staph. aureus (Graber et al., 2009). Samples yielding 2 different bacterial species were considered mixed samples and were, in case of staphylococci, included in the analyses. Samples yielding 3 or more different bacterial species were considered contaminated, and Staphylococcus species from these samples were not further examined.

\section{Inhibition Zone Diameters}

All isolates identified as non-aureus staphylococci and Staph. aureus isolates were submitted to agar disk diffusion testing (Bauer et al., 1966) on Mueller-Hinton agar (i2a, Perols, France) as described previously (Supré et al., 2014). In short, with the InoClic system (i2a), used as described by the manufacturer, a clearly separate colony of the bacteria of interest was picked and suspended in $5 \mathrm{~mL}$ of saline solution, resulting in 0.5 McFarland. The suspension was used for flooding the abovementioned agar plates (i2a) and the excess solution was discarded. Antibiotic-impregnated paper disks were applied with a dispenser.

The panel consisted of fourth-generation cephalosporin (cefquinome, $30 \mu \mathrm{g}$ ), fluoroquinolone (marbofloxacin, $5 \mu \mathrm{g}$ ), the amoxicillin-clavulanic acid combination $(20 / 10 \mu \mathrm{g})$, aminopenicillin (ampicillin, $10 \mu \mathrm{g}$ ), first-generation cephalosporin (cephalonium, $30 \mu \mathrm{g}$ ), macrolide (erythromycin, $15 \mu \mathrm{g}$ ), lincomycin $(15 \mu \mathrm{g})$, oxacillin $(1 \mu \mathrm{g})$, aminoglycoside (neomycin, 30 $\mu \mathrm{g})$, rifaximin $(40 \mu \mathrm{g})$, the sulfonamide-trimethoprim combination $(23.75 / 1.25 \mu \mathrm{g})$, and tetracycline $(30 \mu \mathrm{g})$. Disks were purchased from i2a, except for cefquinome, cephalonium, and rifaximin (Mast Group, Merseyside, UK). After an overnight incubation at $35 \pm 2^{\circ} \mathrm{C}$, plates were read with the SIRscan Micro reader (i2a). The reference strain Staph. aureus ATCC 25923 was used for quality control. Oxacillin was used for detecting methicillin-resistant Staph. aureus, because no structural difference is observed between oxacillin and cefoxitin testing for this bacterium in the Flemish Milk Control Centre laboratory (Lier, Belgium; data not shown). No further investigation was performed to confirm methicillin resistance.

\section{Statistical Analyses}

Before statistical analyses, all outcome and independent variables were transformed into a normal distribution. Because the conventional ways of transformation (e.g., $\log _{10}, \ln$, inverse, square root, quadratic) were not sufficient to obtain normally distributed variables, a 
2-step approach as described by Templeton (2011) was applied. The first step involved transforming the variable into a percentile rank, which resulted in uniformly distributed probabilities. The second step applied the inverse-normal transformation to the results of the first step to form a variable consisting of normally distributed $z$-scores. The mean and standard deviation of the original variable were retained to improve the interpretation of the results.

After normalization of the data, the association between stratum-specific herd-level antimicrobial consumption (i.e., strata 1 to 4 ) and the IZD (in mm) of individual non-aureus staphylococci and Staph. aureus isolates, clustered on the herd level, was evaluated by fitting several separate linear mixed regression models with the IZD of different active substances (cefquinome, marbofloxacin, amoxicillin-clavulanic acid, ampicillin, erythromycin, cephalonium, lincomycin, oxacillin, neomycin, rifaximin, sulfonamide-trimethoprim, and tetracycline) for non-aureus staphylococci and Staph. aureus, respectively, as continuous outcome variables. Per outcome variable, a univariable model was first fit with the stratum-specific herd-level total ATI as continuous independent variable, and then a multivariable model was fit including the stratum-specific herd-level ATI for intramammary therapy of (sub)clinical mastitis, ATI for intramammary dry-cow therapy, and ATI for systemically administered therapy as continuous independent variables.

Second, the association between the IZD of individual non-aureus staphylococci and the IZD of individual Staph. aureus isolates originating from the same herds was studied by fitting univariable linear mixed regression models with the IZD of different active substances (cefquinome, marbofloxacin, amoxicillinclavulanic acid, ampicillin, cephalonium, erythromycin, lincomycin, neomycin, oxacillin, rifaximin, sulfonamidetrimethoprim, and tetracycline) for the non-aureus staphylococci as continuous outcome variables, and the calculated average herd-level IZD of Staph. aureus for each antimicrobial substance as continuous independent variable.

All models contained herd as a random effect to adjust for clustering of isolates within a herd and were fit using PROC MIXED in SAS 9.4 (SAS Institute Inc., Cary, NC). The goodness-of-fit measures included -2 $\times$ log-likelihood, Akaike information criterion, and Bayesian information criterion, using PROC MIXED in SAS 9.4 (SAS Institute Inc.). The conditional Pearson residuals were evaluated graphically and graphed against the normal values and predicted values to check whether the assumptions of normality and homogeneity had been fulfilled, respectively. Also, plots of standardized residuals versus the dependent variables were gen- erated to check whether the assumption of linearity had been fulfilled. No problems were detected. Significance was assessed at $P \leq 0.05$. As multiple associations were investigated, the false discovery rate was calculated using SPSS 24.0 (SPSS Inc./IBM Corp., Chicago, IL).

\section{RESULTS}

\section{Total Antimicrobial Consumption}

The average total ATI was 18.73 DDDA per 1,000 cow-days (Table 1), with a large variation between herds, ranging from 6.28 to 42.13 DDDA. $\beta$-Lactam antimicrobials, both critically important and not critically important, were used in large amounts in the dairy herds. Third- and fourth-generation cephalosporins and penicillins were used on 47, 50, and 51 herds out of the 56 herds, respectively (Table 1). Fourth-generation cephalosporins had the highest ATI (3.57 DDDA per 1,000 cow-days) followed by penicillins (3.23 DDDA) and third-generation cephalosporins (2.95 DDDA).

\section{Classification of Antimicrobials}

Route of Administration and Indication. Antimicrobials administered intramammary had the highest ATI (11.90 DDDA per 1,000 cow-days) followed by systemically administered antimicrobials (6.73 DDDA; Table 1). The use of antimicrobials administered by other routes (e.g., intrauterine) was very low (0.10 DDDA) and therefore not included in this analysis, as mentioned previously. The ATI for intramammary dry-cow therapy was 6.70 DDDA and that for intramammary therapy of (sub)clinical mastitis cases 5.20 DDDA. Dry-cow injectors containing fourth-generation cephalosporins had the highest ATI (1.92 DDDA), followed by first-generation cephalosporins and penicillins (1.68 and 1.30 DDDA, respectively). For the intramammary treatment of (sub)clinical mastitis, the combination first-generation cephalosporins-aminoglycosides had the highest ATI (1.63 DDDA). A large majority of the herds used fourth-generation cephalosporins and the combination of first-generation cephalosporins with aminoglycosides ( $\mathrm{n}=39$ and $\mathrm{n}=36$, respectively) for the treatment of (sub)clinical mastitis.

The average ATI for systemically administered therapy was 6.73 DDDA of which third-generation cephalosporins had the highest ATI (2.66 DDDA), followed by penicillins (1.92 DDDA) and macrolides (0.93 DDDA). Penicillins, third-generation cephalosporins, and macrolides were used systemically on 47,46 , and 40 herds, respectively.

Strata of Antimicrobials. The average ATI of critically important antimicrobials was 6.91 DDDA 
(range 0.00-26.34 DDDA) whereas that for not critically important antimicrobials was 11.83 DDDA (range 0.00-24.76 DDDA; Table 1).

Critically important $\beta$-lactams were used on the majority of the herds, with an average ATI of 6.52 DDDA (range 0.00-26.34 DDDA). Critically important non- $\beta$ lactam antimicrobials, in contrast, were only used on 29 herds, with a low average ATI (0.39 DDDA; range 0.00-2.54 DDDA). A similar distribution was observed for the not critically important antimicrobials, where the average ATI of $\beta$-lactam antimicrobials was higher than that of non- $\beta$-lactam antimicrobials $(10.12$ and 1.71 DDDA, with a range of $0.52-24.76$ and $0.00-6.43$ DDDA, respectively).

\section{Distribution of IZD}

A total of 239 non-aureus staphylococci and 88 Staph. aureus isolates originating from 47 and 33 herds, respectively, were submitted to agar disk diffusion testing. The number of tested isolates per herd varied from 1 to 13 and from 1 to 14 for non-aureus staphylococci and Staph. aureus, respectively. The distribution of IZD of non-aureus staphylococci and Staph. aureus is presented in Table 2. The median IZD of the non-aureus staphylococci and Staph. aureus isolates were 33.0 and 34.0 for cefquinome, 30.0 and 29.0 for marbofloxacin, 39.0 and 40.0 for amoxicillin-clavulanic acid, 34.0 and 40.0 for ampicillin, 35.0 and 40.0 for cephalonium, 28.0 and 27.5 for erythromycin, 30.0 and 31.0 for lincomycin, 24.0 and 21.0 for neomycin, 23.0 and 27.0 for oxacillin, 35.0 and 31.0 for rifaximin, 27.0 and 29.0 for sulfonamide-trimethoprim, and 32.0 and $31.0 \mathrm{~mm}$ for tetracyclines, respectively.

For some isolates, growth was barely inhibited by the presence of the antimicrobial disk (IZD of $6 \mathrm{~mm}$ ) in the agar disk diffusion testing, specifically 10 and 1 isolate(s) for the non-aureus staphylococci-erythromycin and Staph. aureus-erythromycin combination, respectively; 18 and 3 isolates for the non-aureus staphylococcilincomycin and Staph. aureus-lincomycin combination, respectively; 3 and 2 for the non-aureus staphylococcimarbofloxacin and Staph. aureus-marbofloxacin combination, respectively; 5 and 2 isolates for the non-aureus staphylococci-sulfonamide-trimethoprim and Staph. aureus-sulfonamide-trimethoprim, respectively; 4 isolates for the Staph. aureus-oxacillin combination and 2 isolates for the non-aureus staphylococci-tetracyclines combination (Table 2).

Associations Between ATI and IZD. Associations between herd-level ATI (i.e., ATI overall, ATI for intramammary therapy of (sub)clinical mastitis, ATI for intramammary dry-cow therapy, and ATI for systemically administered therapy, respectively) and the
IZD of non-aureus staphylococci and Staph. aureus obtained by Kirby-Bauer disk diffusion are presented in Figure 1.

For non-aureus staphylococci, the ATI for systemic therapy of critically important $\beta$-lactam antimicrobials was negatively associated with the IZD for cefquinome, whereas the ATI for intramammary therapy of (sub) clinical mastitis of critically important $\beta$-lactam antimicrobials was positively associated with the IZD for cefquinome $(P=0.04$ and $P=0.05$, false discovery rate $=84$ and $51 \%$, respectively).

Association Between IZD of Non-Aureus Staphylococcus and Staph. aureus. Only for neomycin was a positive association between the IZD of non-aureus staphylococci and Staph. aureus isolates within the same herd observed $(P<0.01$; false discovery rate: $0.12 \%)$.

\section{DISCUSSION}

Besides precisely quantifying antimicrobial consumption on several commercial dairy herds, we investigated the association between herd-level antimicrobial consumption and IZD, used as a proxy for the presence of antimicrobial resistance, focusing on non-aureus staphylococci (frequently found in milk samples from dairy cows) and Staph. aureus (an important major mastitis pathogen).

The average total ATI in this study was 18.73 DDDA per 1,000 cow-days, which is high compared with the $14.35,3.56,16.05,14.28$, and 4.66 DDDA per 1,000 cow-days obtained in studies in Canada (Saini et al., 2012a), Austria (Obritzhauser et al., 2016), the Netherlands (Kuipers et al., 2016), Argentina (González Pereyra et al., 2015), and New Zealand (McDougall et al., 2016), respectively. Similar to the data obtained in Austria, critically important (for human health) antimicrobials accounted for a large part of the total antimicrobial consumption (Obritzhauser et al., 2016). However, differences in the calculation of antimicrobial consumption hamper a straightforward comparison of the results between the studies (Collineau et al., 2017). The total ATI on the same herds during 2012 and 2013 was 20.78 DDDA per 1,000 cow-days (Stevens et al., 2016), which is slightly higher than described in this article. Because the current study included data obtained as part of a larger 2-yr project, half of the herds included received mastitis management input on a 4- to 6 -weekly basis (intervention group) and 28 herds did not receive mastitis management input but were requested to collect and record the data (control group). In accordance with Kuipers et al. (2016), the expected effect of mastitis management input on a dairy herd's antimicrobial consumption over $2 \mathrm{yr}$ is 


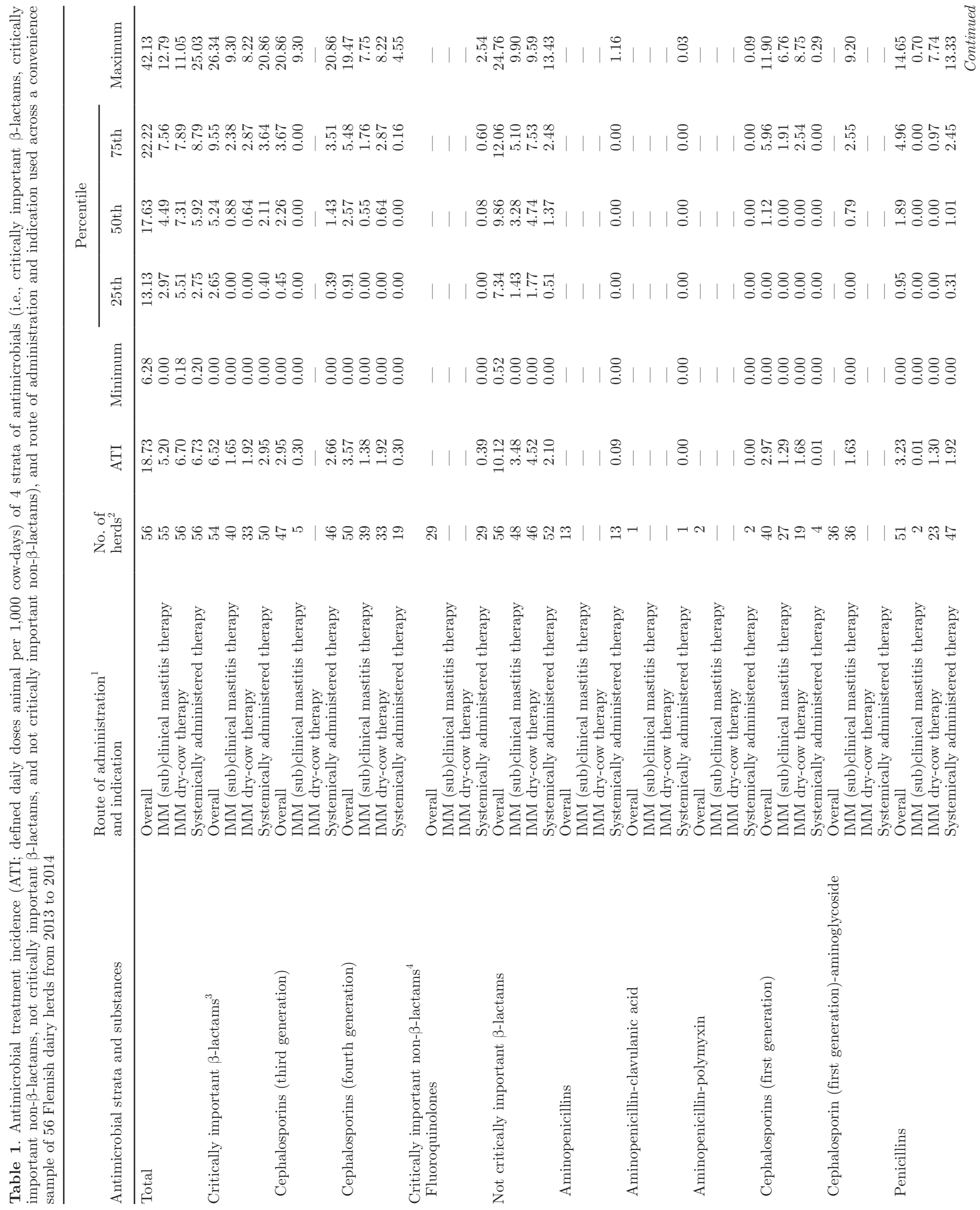


ANTIMICROBIAL CONSUMPTION AND INHIBITION ZONE DIAMETERS OF STAPHYLOCOCCI

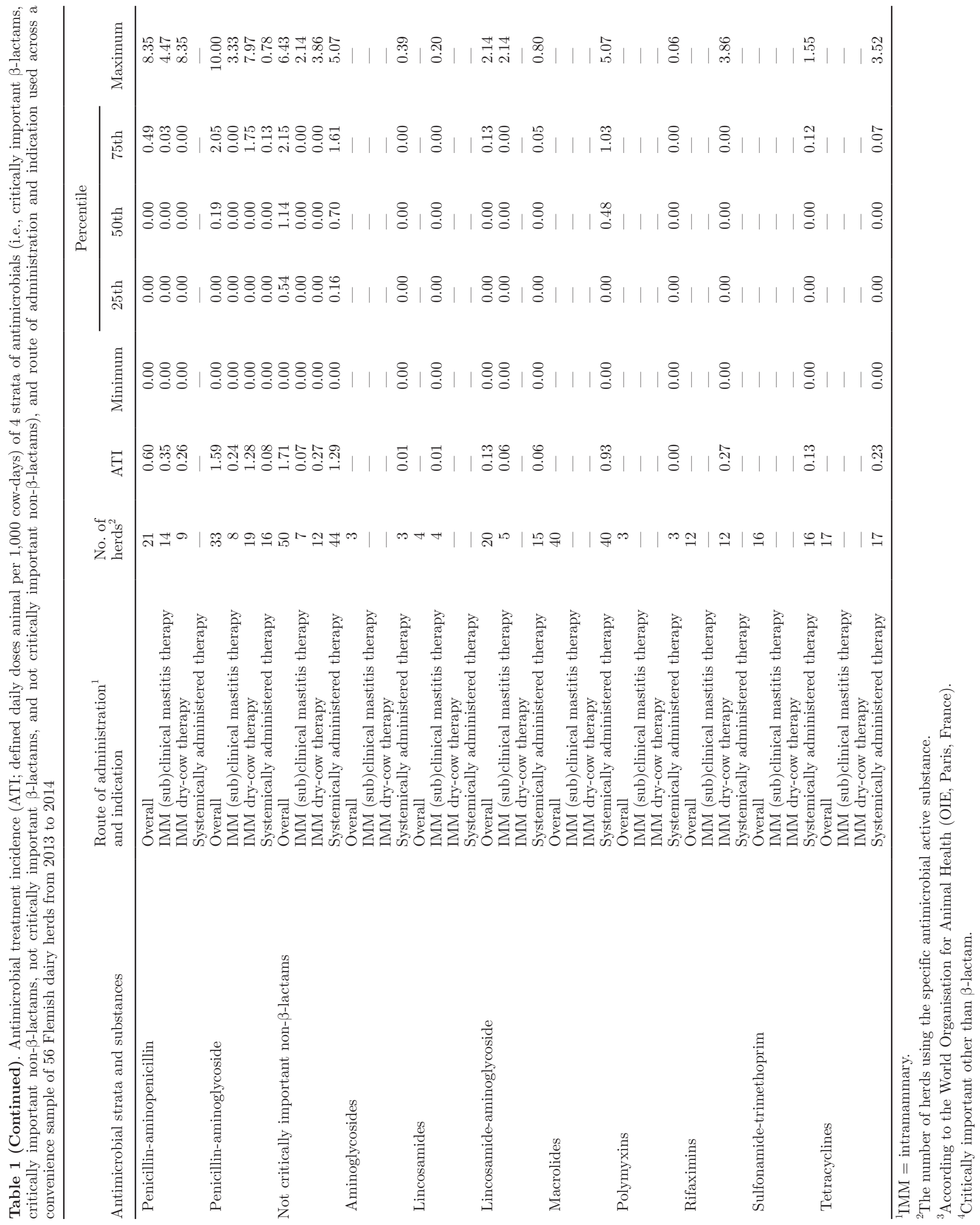




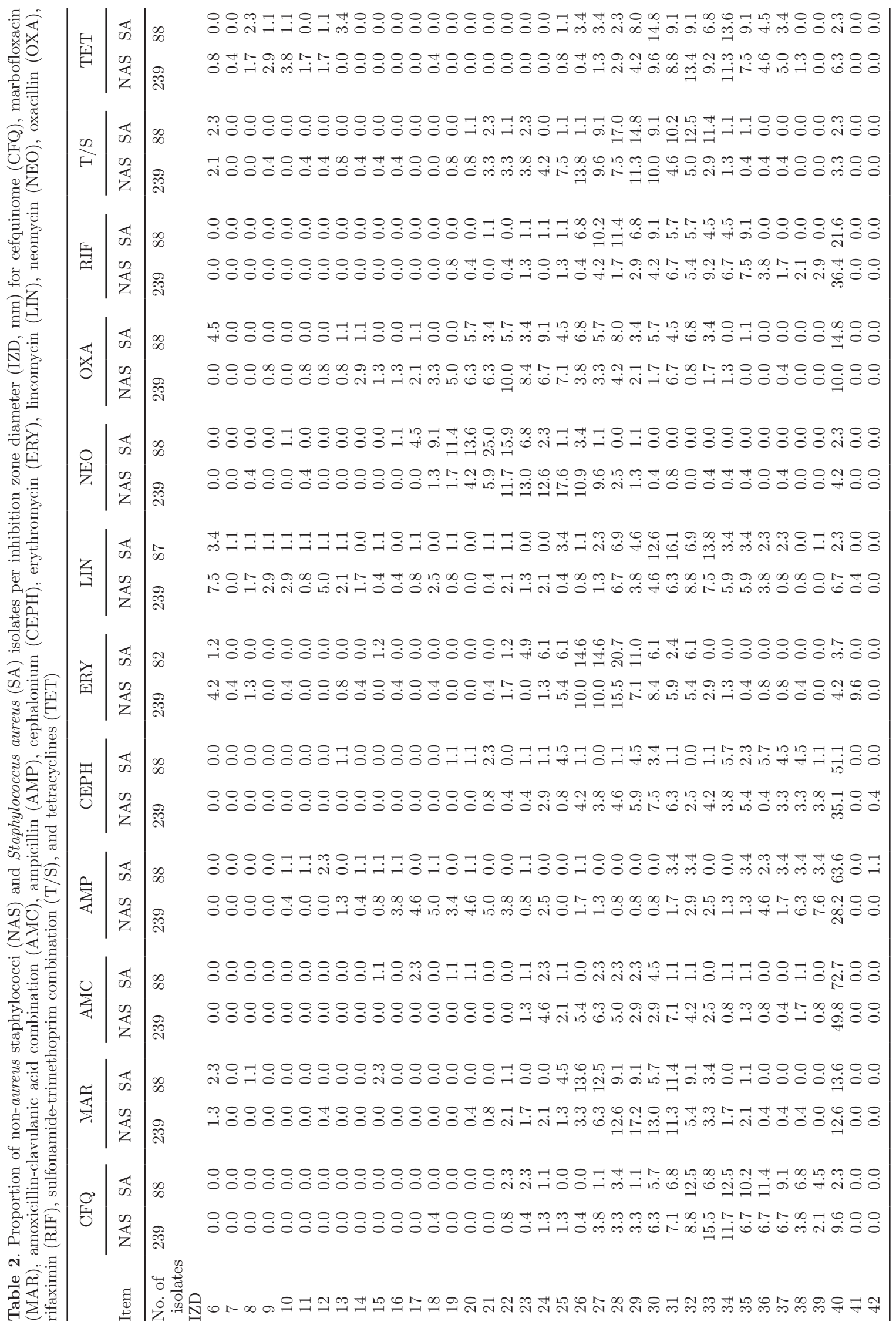


(A)

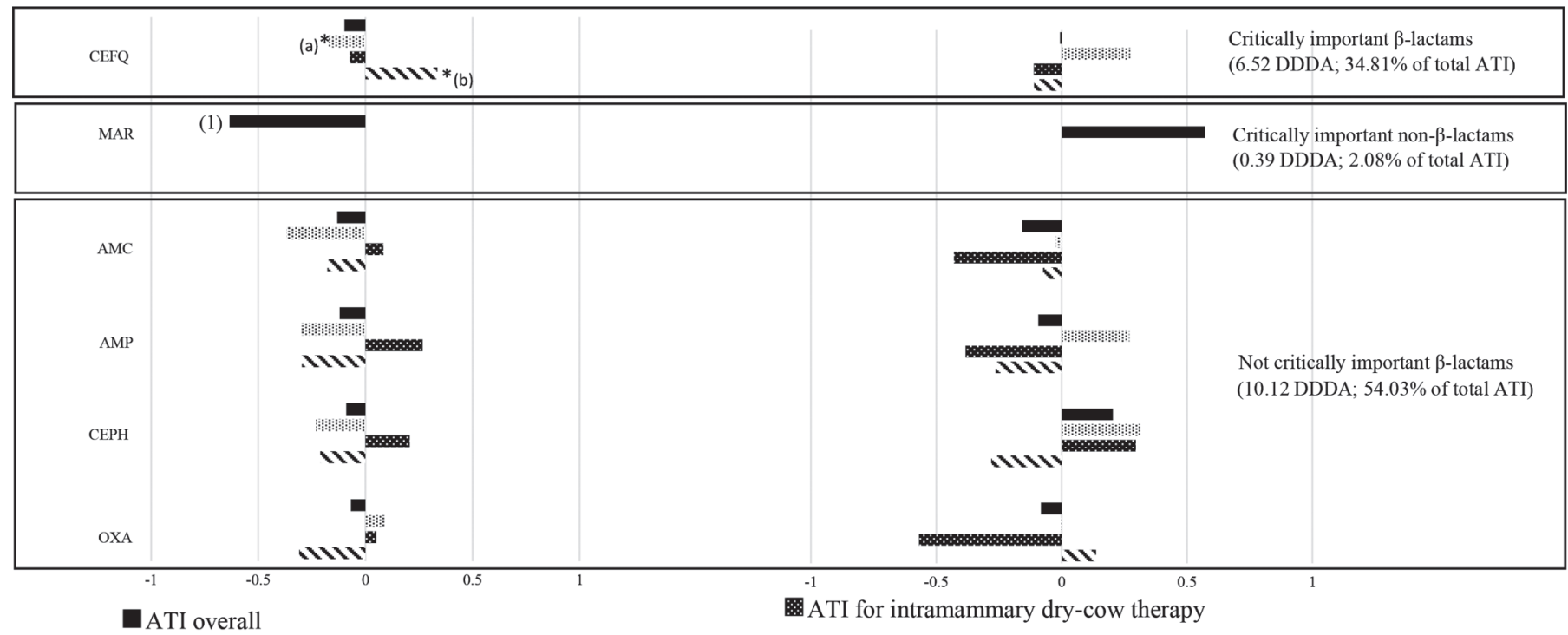

䦶ATI for systemically administered therapy

\$ATI for intramammary therapy of (sub)clinical mastitis

Figure 1. Visualization of associations between inhibition zone diameters (IZD, mm) (CEFQ, cefquinome; MAR, marbofloxacin; AMC, amoxicillin-clavulanic acid; AMP, ampicillin; CEPH, cephalonium; OXA, oxacillin) of (A) non-aureus staphylococci, and (B) Staphylococcus aureus from cows with subclinical mastitis and the herd-level antimicrobial consumption expressed as the antimicrobial treatment incidence (ATI) in defined daily dose animal (DDDA) per 1,000 cow-days for 3 strata of antimicrobials: (top) critically important $\beta$-lactams (third- and fourth-generation cephalosporins), (middle) critically important non- $\beta$-lactams (fluoroquinolones), (bottom) not critically important $\beta$-lactams (aminopenicillins, aminopenicillin-clavulanic acid, aminopenicillin-polymyxin, first-generation cephalosporins, first-generation cephalosporinaminoglycoside, penicillins, penicillin-aminopenicillin, penicillin-aminoglycoside). The horizontal bars represent the $\beta$ (regression factor) originating from univariable models for ATI overall and from multivariable models for herd-level ATI for intramammary therapy of (sub)clinical mastitis, ATI for intramammary dry-cow therapy, and ATI for systemically administered therapy, respectively (see Materials and Methods). *For non-aureus staphylococci, the ATI for systemic therapy of critically important $\beta$-lactam antimicrobials was negatively associated with the IZD for cefquinome $(P=0.04$ with a false-discovery rate of $84 \%)$ and the ATI for intramammary therapy of (sub)clinical mastitis of critically important $\beta$-lactam antimicrobials was positively associated with the IZD for cefquinome $(P=0.05$ with false discovery rate of $51 \%)$. (1) The $\beta$ of "ATI overall" is equal to the $\beta$ of "ATI for systemically administered therapy." In Belgium, Marbofloxacine is only available for systemic use.

limited. The latter assumption appears to be reinforced by the preliminary results obtained in our work in progress. In fact, antimicrobial consumption and changes in antimicrobial consumption on dairy herds seem to be more influenced by changes in mastitis management over time independently of the group to which they were assigned rather than by whether the farm received mastitis management input from the first author. Given that, we concluded that the effect of the farm-based intervention on the results described in this study and their extrapolation is expected to be very limited or nonexistent. Still, we hypothesize that the establishment of a national center of expertise on antimicrobial consumption and resistance in animals (AMCRA, 2014) in 2012 because of political interest in emerging antimicrobial resistance might have played a role as it evolved into several nationwide awareness campaigns targeting farmers and veterinarians, among other projects that were initiated during the time our study was executed.
The Kirby-Bauer disk diffusion method, performed at the Flemish MCC (Lier, Belgium) as part of routine analyses, has a moderate to high diagnostic accuracy and categorical agreement for most udder pathogenantimicrobial combinations (Saini et al., 2011), particularly if plates are read by using the SIRscan Micro (i2a) as was done in this study. Because only a limited number of clinical breakpoints (CLSI) and epidemiological cut off values (EUCAST) are available for mastitiscausing bacteria, we refrained from using cut-off values to stratify isolates into susceptible and resistant populations. Rather, we opted to report IZD and use them as a proxy for antimicrobial resistance when looking for associations with antimicrobial consumption. Still, for most Staph. aureus and non-aureus staphylococciantimicrobial active substance combinations, a bimodal distribution was observed, indicating the presence of acquired resistance in some isolates. Corresponding to the work of Supré et al. (2014), separation of 2 populations based on the curve was less obvious for the Staph. 
aureus-ampicillin combination. This indistinctness was also observed for the non-aureus staphylococci-oxacillin combination.

The negative association between ATI for systemic therapy of critically important $\beta$-lactam antimicrobials and IZD of non-aureus staphylococci for cefquinome might be explained by the (partial) commensal character of non-aureus staphylococci. Because of that commensal character, non-aureus staphylococci are more likely to be exposed to whatever (systemically administered) antimicrobial therapy. The negative association between ATI for systemic therapy of critically important $\beta$-lactam antimicrobials and IZD of nonaureus staphylococci for cefquinome (representing the critically important $\beta$-lactams) is, in this respect, not that surprising and, in fact, confirms selection pressure, because the critically important $\beta$-lactam antimicrobials represent about one-third of the total antimicrobial consumption (6.52 DDDA, of which almost 50\% was used as systemically administered therapy). Still, caution is needed in the interpretation of the data. Although statistically significant associations between IZD and ATI were found in the present study, this does not prove causality. When studying larger data sets with many variables and associations as in the present study, the probability of finding associations by chance increases substantially (Dohoo et al., 1997). Given that, in theory, for every 20 comparisons, a significant difference will be found purely by coincidence without having a real difference (Dewulf et al., 2009), the associations between ATI for systemic therapy of critically important $\beta$-lactam antimicrobials and IZD of cefquinome and the herd-level ATI for intramammary therapy of (sub)clinical mastitis of critically important $\beta$-lactam antimicrobials on the one hand and the IZD of nonaureus staphylococci for cefquinome on the other hand might be due to chance, as reflected by the high false discovery rates. In addition, factors that potentially influence both IZD and herd-level ATI could act as confounders. Still, including herd as a random effect, as done in this study, takes into account the association between isolates within the same herd, and thus controls for any confounding factor at the herd level (Dohoo et al., 2003).

The most common resistance mechanism in staphylococci is the production of $\beta$-lactamase, which results in resistance to penicillin $\mathrm{G}$ and aminopenicillins (Persson Waller et al., 2011; Preethirani et al., 2015; Schmidt et al., 2015). In our results, the trend to resistance, as reflected by a smaller IZD for penicillin G and aminopenicillins, was not pronounced, which is in accordance with findings of Sawant et al. (2009). Still, we are cautious in drawing conclusions about antimicrobial resis- tance in our study, because IZD were used as a proxy for antimicrobial resistance only, and small IZD in the absence of cut-offs and clinical breakpoints are only an indication for the presence of antimicrobial resistance. Moreover, comparison between different studies is likely not legitimate, because different sampling strategies, different susceptibility testing methods (Kirby-Bauer disk diffusion versus broth dilution method), and different interpretive criteria have been used to categorize isolates as either susceptible or resistant. Also, because we did not identify non-aureus staphylococci to the species level and susceptibility to antimicrobials varies between staphylococcal species (Sawant et al., 2009; Bal et al., 2010; Persson Waller et al., 2011; Schmidt et al., 2015; Raspanti et al., 2016), part of the large variation might be explained by differences in IZD between non-aureus staphylococci species.

The positive association in IZD between non-aureus staphylococci and Staph. aureus for neomycin could be explained by the fact that many of the genes encoding neomycin resistance reside on mobile genetic elements, which can be exchanged between bacteria of the same or different species occupying the same ecological niche (Lyon and Skurray, 1987; Lindsay and Holden, 2006).

\section{CONCLUSIONS}

The average ATI on a convenience sample of Flemish dairy herds was high, with a large between-herd variation. Most antimicrobials were used for intramammary therapy. Fourth-generation cephalosporins, critically important for human health, had the highest ATI. A decrease in the antimicrobial susceptibility of non-aureus staphylococci with increasing systemic administration of critically important $\beta$-lactam antimicrobials was suggested. In contrast, the use of critically important $\beta$-lactam antimicrobials for intramammary treatment of (sub)clinical mastitis did not seem to affect the antimicrobial susceptibility of non-aureus staphylococci. The IZD of non-aureus staphylococci and Staph. aureus were positively associated for neomycin.

\section{ACKNOWLEDGMENTS}

All dairy farmers that cooperated are gratefully acknowledged. Special thanks must go to all M-team (Univeristy of Gent) colleagues for helping with herd visits, and to the MCC (Lier, Belgium), the Flemish Farmers Union (Boerenbond, Leuven, Belgium), the Milk Quality Label (IKM-QFL, Brussels, Belgium), and the Belgian Confederation of the Dairy Industry (BCZ, Leuven, Belgium) for partially financing this study. 


\section{REFERENCES}

Acar, J. F., and G. Moulin. 2006. Antimicrobial resistance at farm level. Rev. Sci. Tech. 25:775-792.

AMCRA. 2014. Center of Expertise on Antimicrobial Consumption and Resistance in Animals. Accessed Jan. 10, 2018. http://www .amcra.be/en.

Bal, E. B., S. Bayar, and M. A. Bal. 2010. Antimicrobial susceptibilities of coagulase-negative staphylococci (CNS) and streptococci from bovine subclinical mastitis cases. J. Microbiol. 48:267-274.

Bauer, A. W., W. M. M. Kirby, J. C. Sherris, and M. Turck. 1966. Antibiotic susceptibility testing by a standardized single disk method. Am. J. Clin. Pathol. 45:493-496.

Braem, G., S. De Vliegher, B. Verbist, V. Piessens, E. Van Coillie, L. De Vuyst, and F. Leroy. 2013. Unraveling the microbiota of teat apices of clinically healthy lactating dairy cows, with special emphasis on coagulase-negative staphylococci. J. Dairy Sci. 96:1499-1510.

Chaffer, M., G. Leitner, M. Winkler, A. Glickman, O. Krifucks, E. Ezra, and A. Saran. 1999. Coagulase-negative staphylococci and mammary gland infections in cows. Zentralbl. Veterinarmed. B 46:707-712.

Chantziaras, I., F. Boyen, B. Callens, and J. Dewulf. 2014. Correlation between veterinary antimicrobial use and antimicrobial resistance in food-producing animals: A report on seven countries. J. Antimicrob. Chemother. 69:827-834.

CLSI. 2008. Performance standards for antimicrobial disk and dilution susceptibility tests for bacteria isolated from animals; Approved standard. 3rd ed. CLSI document M31-A3. Clinical and Laboratory Standards Institute, Wayne, PA.

CLSI. 2012. Performance standards for antimicrobial susceptibility testing; Twenty-second informational supplement. CLSI document M100-S22. Clinical and Laboratory Standards Institute, Wayne, PA.

Collineau, L., C. Belloc, K. D. Stark, A. Hemonic, M. Postma, J. Dewulf, and C. Chauvin. 2017. Guidance on the selection of appropriate indicators for quantification of antimicrobial usage in humans and animals. Zoonoses Public Health 64:165-184.

De Visscher, A., S. Piepers, F. Haesebrouck, and S. De Vliegher. 2016. Teat apex colonization with coagulase-negative Staphylococcus species before parturition: Distribution and species-specific risk factors. J. Dairy Sci. 99:1427-1439.

De Visscher, A., S. Piepers, F. Haesebrouck, K. Supre, and S. De Vliegher. 2017. Coagulase-negative Staphylococcus species in bulk milk: Prevalence, distribution, and associated subgroup- and species-specific risk factors. J. Dairy Sci. 100:629-642.

De Visscher, A., K. Supre, F. Haesebrouck, R. N. Zadoks, V. Piessens, E. Van Coillie, S. Piepers, and S. De Vliegher. 2014. Further evidence for the existence of environmental and host-associated species of coagulase-negative staphylococci in dairy cattle. Vet. Microbiol. 172:466-474

De Vliegher, S., L. K. Fox, S. Piepers, S. McDougall, and H. W. Barkema. 2012. Invited review: Mastitis in dairy heifers: Nature of the disease, potential impact, prevention, and control. J. Dairy Sci. 95:1025-1040.

Dewulf, J., D. Maes, A. Van Soom, and A. de Kruif. 2009. How to evaluate and quantify the influence of coincidence on fertility and herd health parameters? A practical overview of frequently used and abused statistical techniques in research and practice. Reprod. Domest. Anim. 44(Suppl. 3):23-30.

Dohoo, I. R., C. Ducrot, C. Fourichon, A. Donald, and D. Hurnik. 1997. An overview of techniques for dealing with large numbers of independent variables in epidemiologic studies. Prev. Vet. Med. 29:221-239.

Dohoo, I. R., S. W. Martin, and H. Stryhn. 2003. Veterinary Epidemiologic Research. VER Inc., Charlottetown, PEI, Canada.

González Pereyra, V., M. Pol, F. Pastorino, and A. Herrero. 2015. Quantification of antimicrobial usage in dairy cows and preweaned calves in Argentina. Prev. Vet. Med. 122:273-279.

Graber, H. U., J. Naskova, E. Studer, T. Kaufmann, M. Kirchhofer, M. Brechbuhl, W. Schaeren, A. Steiner, and C. Fournier. 2009. Masti- tis-related subtypes of bovine Staphylococcus aureus are characterized by different clinical properties. J. Dairy Sci. 92:1442-1451.

Holmes, M. A., and R. Zadoks. 2011. Methicillin resistant S. aureus in human and bovine mastitis. J. Mammary Gland Biol. Neoplasia $16: 373-382$.

Kuipers, A., W. J. Koops, and H. Wemmenhove. 2016. Antibiotic use in dairy herds in the Netherlands from 2005 to 2012. J. Dairy Sci. 99:1632-1648.

Levy, S. B. 1997. Antibiotic resistance: An ecological imbalance. Pages 1-9 in Antibiotic Resistance: Origins, Evolution, Selection and Spread. Vol. 207. D. J. Chadwick and J. Goode, ed. John Wiley \& Sons Ltd., Chichester, West Sussex, UK.

Lindsay, J. A., and M. T. G. Holden. 2006. Understanding the rise of the superbug: Investigation of the evolution and genomic variation of Staphylococcus aureus. Funct. Integr. Genomics 6:186-201.

Lyon, B. R., and R. Skurray. 1987. Antimicrobial resistance of Staphylococcus aureus: Genetic basis. Microbiol. Rev. 51:88-134.

McDougall, S., K. Gohary, A. Bates, and C. Compton. 2016. Antimicrobial usage and resistance. Vol. 2016 Proc. Soc. Dairy Cattle Veterinarians of the NZVA Annu. Conf. VetLearn Foundation, Hamilton, ON, Canada.

Menéndez González, S., A. Steiner, B. Gassner, and G. Regula. 2010. Antimicrobial use in Swiss dairy farms: Quantification and evaluation of data quality. Prev. Vet. Med. 95:50-63.

Mitchell, J. M., M. W. Griffiths, S. A. McEwen, W. B. McNab, and A. J. Yee. 1998. Antimicrobial drug residues in milk and meat: Causes, concerns, prevalence, regulations, tests, and test performance. J. Food Prot. 61:742-756

Nagase, N., A. Sasaki, K. Yamashita, A. Shimizu, Y. Wakita, S. Kitai, and J. Kawano. 2002. Isolation and species distribution of staphylococci from animal and human skin. J. Vet. Med. Sci. 64:245-250.

NMC. 1999. Laboratory Handbook on Bovine Mastitis. Rev. ed. National Mastitis Council (NMC) Inc., Madison, WI.

Obritzhauser, W., M. Trauffler, J. Raith, I. Kopacka, K. Fuchs, and J. Kofer. 2016. Antimicrobial drug use on Austrian dairy farms with special consideration of the use of "highest priority critically important antimicrobials". Berl. Munch. Tierarztl. Wochenschr. 129:185-195.

Oliver, S. P., J. Hogan, B. M. Jayarao, and W. E. Owens. 2004. Microbiological procedures for the diagnosis of bovine udder infection and determination of milk quality. Pages $1-8$ in Proc. 4th. National Mastitis Council Annu. Mtg. National Mastitis Council Inc., Verona, WI.

Otto, M. 2013. Coagulase-negative staphylococci as reservoirs of genes facilitating MRSA infection. BioEssays 35:4-11.

Owens, W. E., and J. L. Watts. 1988. Antimicrobial susceptibility and beta-lactamase testing of staphylococci isolated from dairy herds. J. Dairy Sci. 71:1934-1939.

Paduch, J. H., and V. Kroemker. 2011. Colonization of the teat skin and the teat canal by mastitis pathogens in dairy cattle. Tieraerztl. Prax. Ausg. G Grosstiere Nutztiere 39:71-76.

Persson Waller, K., A. Aspán, A. Nyman, Y. Persson, and U. Grönlund Andersson. 2011. CNS species and antimicrobial resistance in clinical and subclinical bovine mastitis. Vet. Microbiol. 152:112-116.

Piepers, S., L. De Meulemeester, A. de Kruif, G. Opsomer, H. W. Barkema, and S. De Vliegher. 2007. Prevalence and distribution of mastitis pathogens in subclinically infected dairy cows in Flanders, Belgium. J. Dairy Res. 74:478-483.

Piepers, S., K. Peeters, G. Opsomer, H. W. Barkema, K. Frankena, and S. De Vliegher. 2011. Pathogen group specific risk factors at herd, heifer and quarter levels for intramammary infections in early lactating dairy heifers. Prev. Vet. Med. 99:91-101.

Piessens, V., S. De Vliegher, B. Verbist, G. Braem, A. Van Nuffel, L. De Vuyst, M. Heyndrickx, and E. Van Coillie. 2012. Intra-species diversity and epidemiology varies among coagulase-negative Staphylococcus species causing bovine intramammary infections. Vet. Microbiol. 155:62-71.

Pol, M., and P. L. Ruegg. 2007a. Relationship between antimicrobial drug usage and antimicrobial susceptibility of gram-positive mastitis pathogens. J. Dairy Sci. 90:262-273. 
Pol, M., and P. L. Ruegg. 2007b. Treatment practices and quantification of antimicrobial drug usage in conventional and organic dairy farms in Wisconsin. J. Dairy Sci. 90:249-261.

Preethirani, P. L., S. Isloor, S. Sundareshan, V. Nuthanalakshmi, K. Deepthikiran, A. Y. Sinha, D. Rathnamma, K. Nithin Prabhu, R. Sharada, T. K. Mukkur, and N. R. Hegde. 2015. Isolation, biochemical and molecular identification, and in vitro antimicrobial resistance patterns of bacteria isolated from bubaline subclinical mastitis in South India. PLoS One 10:e0142717.

Rajala-Schultz, P. J., K. L. Smith, J. S. Hogan, and B. C. Love. 2004. Antimicrobial susceptibility of mastitis pathogens from first lactation and older cows. Vet. Microbiol. 102:33-42.

Raspanti, C. G., C. C. Bonetto, C. Vissio, M. S. Pellegrino, E. B. Reinoso, S. A. Dieser, C. I. Bogni, A. J. Larriestra, and L. M. Odierno. 2016. Prevalence and antibiotic susceptibility of coagulasenegative Staphylococcus species from bovine subclinical mastitis in dairy herds in the central region of Argentina. Rev. Argent. Microbiol. 48:50-56.

Saini, V., J. T. McClure, D. Leger, S. Dufour, A. G. Sheldon, D. T. Scholl, and H. W. Barkema. 2012a. Antimicrobial use on Canadian dairy farms. J. Dairy Sci. 95:1209-1221.

Saini, V., J. T. McClure, D. T. Scholl, T. J. DeVries, and H. W. Barkema. 2012b. Herd-level association between antimicrobial use and antimicrobial resistance in bovine mastitis Staphylococcus aureus isolates on Canadian dairy farms. J. Dairy Sci. 95:1921-1929.

Saini, V., R. G. M. O. Riekerink, J. T. McClure, and H. W. Barkema. 2011. Diagnostic accuracy assessment of Sensititre and agar disk diffusion for determining antimicrobial resistance profiles of bovine clinical mastitis pathogens. J. Clin. Microbiol. 49:1568-1577.

Sawant, A. A., B. E. Gillespie, and S. P. Oliver. 2009. Antimicrobial susceptibility of coagulase-negative Staphylococcus species isolated from bovine milk. Vet. Microbiol. 134:73-81.

Schmidt, T., M. M. Kock, and M. M. Ehlers. 2015. Diversity and antimicrobial susceptibility profiling of staphylococci isolated from bovine mastitis cases and close human contacts. J. Dairy Sci. 98:6256-6269.

Silbergeld, E. K., J. Graham, and L. B. Price. 2008. Industrial food animal production, antimicrobial resistance, and human health. Annu. Rev. Public Health 29:151-169.
Stevens, M., S. Piepers, K. Supre, J. Dewulf, and S. De Vliegher. 2016. Quantification of antimicrobial consumption in adult cattle on dairy herds in Flanders, Belgium, and associations with udder health, milk quality, and production performance. J. Dairy Sci. 99:2118-2130.

Supré, K., K. Lommelen, and L. De Meulemeester. 2014. Antimicrobial susceptibility and distribution of inhibition zone diameters of bovine mastitis pathogens in Flanders, Belgium. Vet. Microbiol. 171:374-381.

Sztachańska, M., W. Baranski, T. Janowski, J. Pogorzelska, and S. Zdunczyk. 2016. Prevalence and etiological agents of subclinical mastitis at the end of lactation in nine dairy herds in North-East Poland. Pol. J. Vet. Sci. 19:119-124.

Taponen, S., and S. Pyorala. 2009. Coagulase-negative staphylococci as cause of bovine mastitis-Not so different from Staphylococcus aureus? Vet. Microbiol. 134:29-36.

Templeton, G. F. 2011. A two-step approach for transforming continuous variables to normal: Implications and recommendations for IS research. Comm. Assoc. Inform. Syst. 28:41-58.

Traub, W. H., and B. Leonhard. 1994. Agar disk diffusion (BauerKirby) tests with various fastidious and nonfastidious reference (ATCC) strains: Comparison of several agar media. Chemotherapy 40:374-383.

Vanderhaeghen, W., S. Piepers, F. Leroy, E. Van Coillie, F. Haesebrouck, and S. De Vliegher. 2014. Invited review: Effect, persistence, and virulence of coagulase-negative Staphylococcus species associated with ruminant udder health. J. Dairy Sci. 97:5275-5293.

Vitali, L. A., D. Petrelli, A. Lamikanra, M. Prenna, and E. O. Akinkunmi. 2014. Diversity of antibiotic resistance genes and staphylococcal cassette chromosome mec elements in faecal isolates of coagulase-negative staphylococci from Nigeria. BMC Microbiol. 14:106.

White, D. G., R. J. Harmon, J. E. Matos, and B. E. Langlois. 1989 Isolation and identification of coagulase-negative Staphylococcus species from bovine body sites and streak canals of nulliparous heifers. J. Dairy Sci. 72:1886-1892. 